\title{
Evaluation of the psychometric properties of the nursing students' attitudes toward mental health nursing and consumers instrument
}

\author{
Marcos Hirata Soares ${ }^{* 1}$, Margarita Antonia Villar Luís ${ }^{2}$ \\ ${ }^{1}$ Department of Nursing, State University of Londrina, Parana State, Brazil \\ ${ }^{2}$ Department of Psychiatric Nursing and Human Sciences of the Ribeirão Preto, College of Nursing, USP, Sao Paulo State, Brazil
}

Received: March 1, 2016

DOI: $10.5430 /$ jnep.v6n10p122

\author{
Accepted: June 1, 2016 \\ Online Published: June 14, 2016
}

URL: http://dx.doi.org/10.5430/jnep.v6n10p122

\begin{abstract}
The present study aimed to evaluate the psychometric properties of the instrument "Nursing Students' Attitudes Toward Mental Health Nursing and Consumers" for use among nursing students in Brazil. The subjects were 91.3\% female and 8.7\% male and their ages ranged from 18 to 58 years, with a mean (M) of 21.9 years and a standard deviation (sd) of 3.88 years. The study included students from the undergraduate course in nursing of five higher public education institutions, with a total of 393 students. Of these, 365 answered the questionnaires at their two application times, resulting in $92.87 \%$ full participation in the study. Regarding the school year, 23.9\% were from 2013 and 76.1\% from 2014. The data were entered into the Statistical Package for the Social Sciences (SPSS) program, v.21, to be analyzed by descriptive exploratory analysis, correlations analysis, means comparison and Principal Component Analysis (PCA) with the exploratory factor analysis technique and oblique rotation. Convergent validity was used as the form of validation, using the Authoritarianism and Minority View dimensions of the Opinions about Mental Illness (OMI) Scale. After generating the structure of the instrument to be tested, the Confirmatory Factor Analysis technique (CFA) was used, through the Structural Equation Modeling technique (SEM), with AMOS/SPSS, v.22, to identify and specify the model, through Maximum Likelihood (ML) estimation. The fit was given by the $\chi^{2}$ model, and the absolute, incremental and parsimonious fit indices.
\end{abstract}

Key Words: Nursing students, Mental health, Psychometric properties

\section{INTRODUCTION}

The end of the 1950s is marked by the beginning of the development of several instruments to measure opinions, beliefs and attitudes towards mental illness and the mentally ill, an interest that has lasted up to the present day. In this study the research is focused on the instruments directed toward nursing students in the mental health area. The literature review identified studies performed in Australia on the low preference of nurses to work the mental health area ${ }^{[1-4]}$ and initiatives for the application of recruitment and training methods for the practice in mental health care. ${ }^{[5]}$

There is a lack, in Brazil, of recent studies on the subject using instruments, highlighting one conducted in partnership with researchers from Peru and Chile, on the profile of attitudes of nursing students towards mental disorders. ${ }^{[6]}$ However, qualitative studies ${ }^{[7]}$ show the presence of negative

*Correspondence: Marcos Hirata Soares; Email: mhirata@uel.br; Address: State University of Londrina, Department of Nursing, Av. Robert Koch, 60 Vila Operária, 86038-390, Londrina, Paraná, Brazil. 
attitudes of students, both at the beginning and the end of the course, towards mental illness and attitudes ranging from authoritarianism to benevolence. These indications may help comprehend the low attraction and preference for Psychiatric/Mental Health Nursing in relation to the other Nursing specialties.

The present study aimed to evaluate the psychometric properties of the instrument "Nursing Students' Attitudes Toward Mental Health Nursing and Consumers" for use among Nursing students in Brazil.

It is understood that the evaluation of the psychometric properties of the instrument and the testing and improvement of an instrument that evaluates learning in mental health, will allow the effect of the variables involved to be measured, explain the problem and predict future events, as well as allow generalizations from its application to other subjects, contexts and socio-political and historical moments.

About the "Nursing Students' Attitudes Toward Mental Health Nursing and Consumers" instrument, this instrument originates from a set of theories, experience of care practice, teaching and evaluation of the teaching-learning process of the student in mental health, incorporated by Nursing over decades. The main knowledge that influenced the first theories of nursing in the 1950 s to 1970 s, relate to the psychodynamic and interpersonal humanistic principles of Sigmund Freud, Harry Sullivan and Carl R. Rogers, as well as Hildegard Peplau and Joyce Travelbee, ${ }^{[8]}$ authors whose concepts also underlie the theory and practice of Brazilian psychiatric nursing education.

The purpose of the instrument is to measure the attitudes of nursing students towards mental health patients and nursing. It was originally applied with 687 nursing students who attended the mental health discipline in 21 mental health services in Victoria, Australia. ${ }^{[1]}$ The application occurs in two stages: at the beginning of the mental health discipline, the instrument is applied with 24 sentences; the second measurement takes place at the end of the discipline, with the addition of 14 questions to the instrument (items 25 to 39) to evaluate the influence of the clinical internship on the student.

The instrument consists of 24 statements (section A) and four demographic questions (section B), which include gender, university, year of the course and information about a career in mental health nursing. The statements qualify three areas of perceptions and experiences of the students: 1) preparation for the field of mental health; 2) attitudes towards mental disorder and users of mental health services; 3 ) attitudes towards mental health nursing, including career preferences.
On the final day of the clinical internship, items 25 to 39, assess the availability of the nursing staff toward the student, in order to evaluate the influence or the contribution of the clinical internship on the formation and future choice of career. Students respond to each of the statements using a Likert scale with 7 points, where $1=$ totally disagree, $2=$ strongly disagree, 3 = disagree, $4=$ neither agree nor disagree, $5=$ agree, $6=$ strongly agree, $7=$ totally agree

The domains and internal consistency of the original version of the instrument ${ }^{[1]}$ are as follows:

- Preparedness for the mental health field (PMHF), composed by sentences 1, 4, 7 and 10. Higher scores represent a greater sense of preparedness. Alfa $=0.72$;

- Knowledge about mental health (KMI), composed by sentences 9, 18, 19 and 23. Higher scores represent a more informed attitude. Alfa $=0.56$;

- Negative stereotypes (NS), composed by sentences 8, 21 and 24. Lower scores indicate less stereotyped beliefs. Alfa $=0.51$;

- Future career (FC), composed by sentences 6 and 12 . Higher scores represent a greater desire to pursue a career in mental health nursing. Alfa $=0.92$;

- Course effectiveness (CE), composed by sentences 14 to 17 . Higher scores represent the degree to which the Nursing university courses have prepared the students for the various nursing practice areas. Alfa $=0.55$;

- Anxiety surrounding mental illness (ASMI), composed by items 3 and 5 (both with reversed score) and item 22 (higher scores represent lower levels of anxiety). Alfa = 0.67;

- Valuable contributions (VC), composed by items 2, 11 and 20. Higher scores represent a stronger belief that psychiatric nurses provide a valuable service to users, the community and students' nursing careers. Alfa $=$ 0.67 .

- Post-clinical internship (POS): Composed by items 25 to 39. Assesses the availability of nursing staff for the students. Higher scores indicate widespread availability of staff and a perceived level of clinical skill in the team involved with the students. Alfa $=0.84$.

Due to the scores observed, it was considered important to invest in the evaluation and testing of this instrument, considering its focus is on mental health learning and that it could allow measurement of the effect of the variables involved. This will enable the explanation of the problem and even the forecasting of situations. The aim was to improve the instrument and enable its widespread use, with its application to other subjects, contexts and socio-political and historical moments. 


\section{MATERIAL AND METHODS}

This is a methodological study to evaluate the psychometric properties of the subjectively constructed measuring instrument, with undergraduate nursing students experiencing the curricular mental health discipline, in which the institution, offered to students, teachers with solid training in the mental health area and practical activities in non-hospitalization services. The research project was approved by the Research Ethics Committee at the Londrina State University (CAAE No. 12795513.6.0000.5231), as well as the HEIs participating in the study and the author of the instrument.

In the present study, as the pre-test, the instrument was applied with a group of 30 students to ensure there was comprehension. The results indicated the need for adaptation to the local culture. The cultural adaptation is considered to be a process oriented toward measuring a similar phenomenon in a different culture; it is essentially the production of an equivalent instrument adapted to another culture. ${ }^{[9]}$ This process was conducted ${ }^{[10]}$ and resulted in the need to make changes in the content of the original version. Due to the scores observed, at this stage it was considered relevant to invest in the evaluation and testing of the instrument.

Several authors suggest procedures for conducting evaluation studies of the cultural adaptation and psychometric properties of instruments. The choosed reference ${ }^{[11]}$ describes the stages of conceptual, item, semantic, operational and measurement equivalence. The first three steps are cited in study, ${ }^{[10]}$ while the final step is equivalent to the data analysis procedures using statistical packages and methods of analysis of the indicated data.

\section{Data analysis}

The standardized Cronbach's alpha coefficient, the mean of between items correlation and inter-item correlation were calculated. Convergent validity was used as the form of validation, using the Authoritarianism and Minority View dimensions of the Opinions about Mental Illness (OMI) Scale, ${ }^{[12,13]}$ validated in Brazil, aiming for a correlation coefficient greater than 0.45 , with the domains of the instrument called Authoritarianism and Minority Vision.

The data were entered into the Statistical Package for the Social Sciences (SPSS) program, v.21, to be analyzed by descriptive exploratory analysis, correlations analysis, means comparison and Principal Component Analysis (PCA) with the exploratory factor analysis technique and oblique Oblimin rotation. After generating the structure of the instrument to be tested, the Confirmatory Factor Analysis technique (CFA) was used, through the Structural Equation Modeling technique (SEM), with AMOS/SPSS, v.22, to identify and specify the model, through Maximum Likelihood (ML) estimation. The fit was given by the $\chi^{2}$ model, and the absolute, incremental and parsimonious fit indices, as the RMSEA, CFI, GFI, PGFI. ${ }^{[14]}$

\section{Results}

The study included students from the 2nd, 3rd and 4th grades of the undergraduate course in nursing of five public education institutions, two in the capital of São Paulo, one in the state, one in the capital of Paraná and one in the state, with a total of 393 students. Of these, 365 answered the questionnaires at their two application times, resulting in $92.87 \%$ full participation in the study.

The subjects were $91.3 \%$ female and $8.7 \%$ male and their ages ranged from 18 to 58 years, with a mean (M) of 21.9 years and a standard deviation (sd) of 3.88 years. The timetable of theoretical classes ranged from 36 to 71 hours of classes, $\mathrm{M}=52$ hours; $\mathrm{sd}=13.64$ and the practical timetable, ranged from 76 to 100 hours of classes, $\mathrm{M}=91$ and $\mathrm{sd}=5.22$. The length of the course varied depending on the schedule and hours of classes of each Higher Education Institution (HEI). Thus, this variable ranged between 29 and 136 days, $\mathrm{M}=77.63, \mathrm{sd}=39.64$.

Regarding the school year, 23.9\% were from 2013 and $76.1 \%$ from 2014. Regarding the grade, $37.9 \%$ were enrolled in 2nd grade, $35.4 \%$ in the 3 rd grade and $26.7 \%$ in the 4 th grade. Concerning the location, $16.8 \%$ were from the HEI within the state of Paraná (PR) and 9.9\% from the HEI of the capital. For the rest, $52.4 \%$ of the sample was obtained from the two HEIs of the capital of São Paulo (SP) and 20.9\% from the HEI within the state.

\section{Principal Component Analysis (PCA) and Reliability}

The first step was to conduct an exploratory factor analysis using the extraction by principal components technique. The initial criterion of commonality $<0.4$ was established for deleting the item from the instrument, a minimum of 0.7 for the Kaiser-Meyer-Olkin index (KMO) for the sample to be deemed adequate and $p<.01$ for Bartlett's test of sphericity. The sample initially presented a KMO value of 0.87 , explained variance of $63.90 \%$, with 9 extracted components and $p<.001$ for Bartlett's test, as well as all of the items having commonalities with values greater than 0.40 . The extraction by principal component technique was used and oblique Oblimin rotation.

In a second analysis, the removal of 7 components was established, to try to follow the original structure of the instrument. With this, there was a decrease in the values of the commonalities of 6 factors, leading to the application of the criteria 
and the performance of a new analysis. The third analysis resulted in 29 items, of the 35 determined in the cross-cultural adaptation, with commonalities $>0.4$. After extraction of the factors, a KMO index for the sample of 0.88 was obtained, with explained variance of $66.07 \%$ for 7 components and $p$ $<.001$ for Bartlett's test (29 item version). When analyzing the reliability of the domains of the scale, it was noticed that there were 03 domains with low values $(<0.70)$. Therefore, the withdrawal of the NS (items 08, 21, 24), and KMI (09, 19, 23) domains was carried out, however, the VC domain was maintained as it presented a value of 0.69 , very close to the acceptable minimum. The new version, with 23 items, presented an explained variance of $67.30 \%, \mathrm{KMO}$ index of 0.89 and Bartlett's test with $p<.001$, with five principle components extracted and the new standardized reliability score of the VC subscale being 0.71 .

In Table 1, it should be noted that the correlations with the domains of the OMI scale, despite being statistically significant, were weak, not guaranteeing the assumption that the evaluated construct converged with that analyzed by the OMI scale.

Table 1. Matrix of correlations between the domains and convergent validation in the 29 item version of the instrument

\begin{tabular}{|c|c|c|c|c|c|c|c|c|}
\hline & Authoritarianism & Minority view & PMHF & NS & KMI & FC & VC & POS \\
\hline $\begin{array}{l}\text { Authoritarianism } \\
p \text { value }\end{array}$ & $* * *$ & & & & & & & \\
\hline $\begin{array}{l}\text { Minority view } \\
p \text { value }\end{array}$ & $\begin{array}{l}.403^{* *} \\
.000\end{array}$ & $* * *$ & & & & & & \\
\hline $\begin{array}{l}\text { PMHF } \\
p \text { value }\end{array}$ & $\begin{array}{l}-.180^{* *} \\
.001\end{array}$ & $\begin{array}{l}-.088 \\
.094\end{array}$ & $* * *$ & & & & & \\
\hline $\begin{array}{l}\text { NS } \\
p \text { value }\end{array}$ & $\begin{array}{l}.081 \\
.124\end{array}$ & $\begin{array}{l}.084 \\
.108\end{array}$ & $\begin{array}{l}-.250^{* *} \\
.000\end{array}$ & $* * *$ & & & & \\
\hline $\begin{array}{l}\text { KMI } \\
p \text { value }\end{array}$ & $\begin{array}{l}-.160^{* *} \\
.002\end{array}$ & $\begin{array}{l}-.130^{*} \\
.013\end{array}$ & $\begin{array}{l}.195^{* *} \\
.000\end{array}$ & $\begin{array}{l}-.207^{* *} \\
.000\end{array}$ & $* * *$ & & & \\
\hline $\begin{array}{l}\text { FC } \\
p \text { value }\end{array}$ & $\begin{array}{l}-.082 \\
.116\end{array}$ & $\begin{array}{l}-.152^{* *} \\
.004\end{array}$ & $\begin{array}{l}.341^{* *} \\
.000\end{array}$ & $\begin{array}{l}-.159^{* *} \\
.002\end{array}$ & $\begin{array}{l}.046 \\
.378\end{array}$ & $* * *$ & & \\
\hline $\begin{array}{l}\mathrm{VC} \\
p \text { value }\end{array}$ & $\begin{array}{l}-.049 \\
.353\end{array}$ & $\begin{array}{l}-.065 \\
.212\end{array}$ & $\begin{array}{l}.422^{* *} \\
.000\end{array}$ & $\begin{array}{l}-.005 \\
.923\end{array}$ & $\begin{array}{l}.376^{* *} \\
.000\end{array}$ & $\begin{array}{l}.151^{* *} \\
.004\end{array}$ & $* * *$ & \\
\hline $\begin{array}{l}\text { POS } \\
p \text { value }\end{array}$ & $\begin{array}{l}-.112^{*} \\
.033\end{array}$ & $\begin{array}{l}-.008 \\
.885\end{array}$ & $\begin{array}{l}.521^{* *} \\
.000\end{array}$ & $\begin{array}{l}-.123^{*} \\
.018\end{array}$ & $\begin{array}{l}.190^{* *} \\
.000\end{array}$ & $\begin{array}{l}.201^{* *} \\
.000\end{array}$ & $\begin{array}{l}.448^{* *} \\
.000\end{array}$ & $* * *$ \\
\hline
\end{tabular}

Correlation is significant at level .01 (two-tailed). **; Correlation is significant at level .05 (two-tailed). *

Table 2. Standardized Cronbach's alpha coefficient scores for the domains in the proposed versions of the instrument

\begin{tabular}{llll}
\hline Domain/ACP & $\begin{array}{l}\mathbf{1}^{\text {st }} \text { PCA- } \\
\mathbf{3 5} \text { items }\end{array}$ & $\begin{array}{l}\mathbf{2}^{\text {nd }} \mathbf{P C A}- \\
\mathbf{2 9} \text { items }\end{array}$ & $\begin{array}{l}3^{\text {rd }} \text { PCA- } \\
\mathbf{2 3} \text { items }\end{array}$ \\
\hline Overall & .87 & .89 & .91 \\
PMHF & .77 & .81 & .81 \\
NS & .64 & .64 & $* * *$ \\
KMI & .41 & .42 & $* * *$ \\
FC & .95 & .95 & .95 \\
VC & .69 & .69 & .71 \\
POS & .88 & .89 & .89 \\
\hline
\end{tabular}

As shown in Table 2, the standardized alpha score improved in the 23 item instrument, meaning that this version is more consistent than the original, of 35 items. Upon completion of the PCA procedure and based on a new discussion about the

Published by Sciedu Press relationship between the items and their domains, the CFA step was initiated, attempting to test the theoretical model, considering that the original instrument has not been proved valid for the Brazilian reality, with there being no proof of its validity in the original country. This procedure was necessary so that the changes made would sustain the new instrument and help the creation of a theoretical model from the SEM for the proposed new instrument.

This new model was composed as follows:

(1) PMHF-R("R" of revised) domain: items 01, 04, 07, 10, 22 and 28. Represents the Preparedness for the mental health field. Higher scores represent a strong feeling of preparedness to practice in the mental health area. Mean $(\mathrm{M})=4.92$, Standard deviation $(\mathrm{sd})=0.96$.

(2) VC domain: items 02, 11 and 20. Represents Valuable 
Contributions: Higher scores represent a strong belief that psychiatric nurses provide a valuable service to users, the community and the nursing careers of students. $\mathrm{M}=6.27, \mathrm{sd}=0.88$.

(3) TS Domain: items 26, 31, 32 and 36. Represents Teacher Supervision. Higher scores represent a positive perception of the teacher supervision in the practical activities. $\mathrm{M}=6.11, \mathrm{sd}=1.07$.

(4) ENS domain: items 27, 33, 34, 35, 37, 38 and 39. Represents the Evaluation of the Nursing Staff. Represents the evaluation regarding the attitude of the nursing staff towards the students and patients. $\mathrm{M}=4.95, \mathrm{sd}=1.49$.

(5) FC domain: items 06, and 12. Represents the Future Career. Higher scores represent a strong desire to pursue a career in mental health nursing. $\mathrm{M}=2.79, \mathrm{sd}=$ 1.83 .

From the model generated in the PCA, the CFA procedure was applied for the verification of the fit of its factor structure. The measurement model of five factors of the scale was adjusted by maximum likelihood (ML) and is presented in Figure 1. Initially, the model needed to be revised, with correlations being attributed between items 22 and 36, 26 and 27, and 36 with the FC domain, so that the model had an adequate fit, with item 30 also being removed to improve the fit of the model.

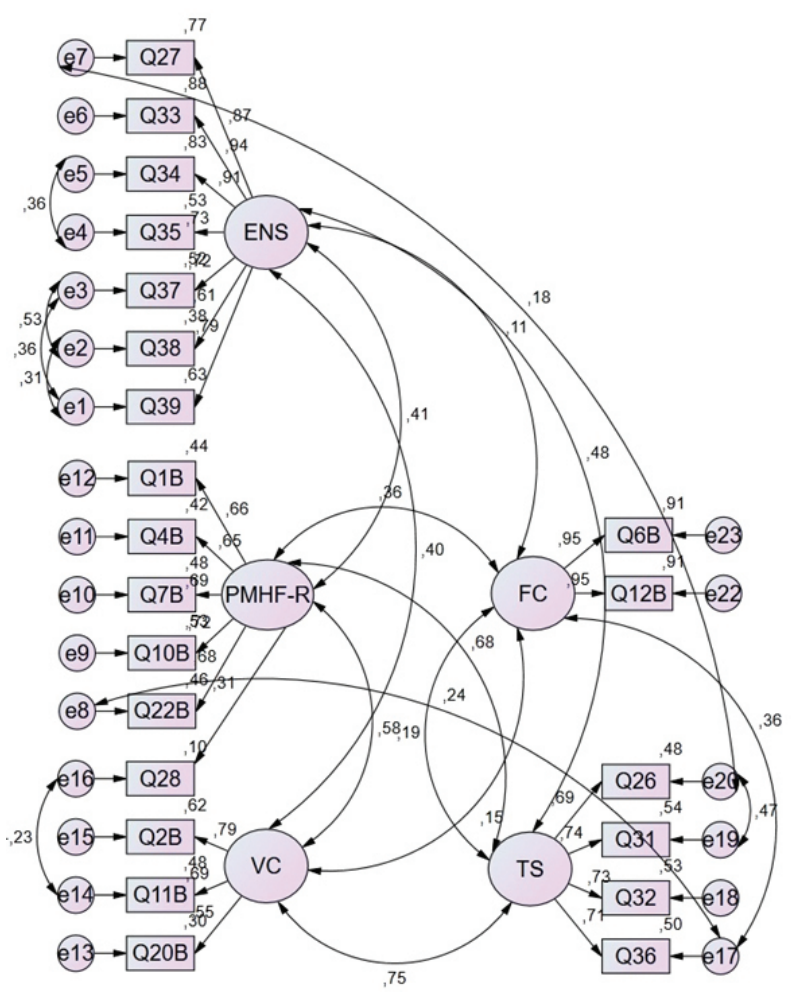

Figure 1. Theoretical model validated by SEM
Only the FC and VC domains remained identical to the original instrument. Thus, there were two new domains that were the result of the changes to the POS domain, called by the author ENS (which is an evaluation of the attitude of the nursing staff in relation to the students and patients) and TS (which is their perception in relation to the teacher supervision of their practical activities), with two items added to the PMHF domain, causing this to be called PMHF-R symbolizing the restructured form of this domain. The validated model presented in Figure 1 indicated significant correlations.

The absolute fit indices, such as GFI (Goodness-of-Fit Index), AGFI (Adjusted Goodness-of-Fit Index) and RMSEA (Root Mean Square Error of Approximation) showed values of $0.90,0.87,0.05$, respectively, the incremental fit indices (NFI (Bentler-Bonnet Normed Fit Index), CFI (Comparative Fit Index)) were 0.91 and 0.95 , and the PGFI (Parsimony Goodness-of-Fit Index) was 0.68, allowing the researchers to accept the theoretical model as valid. The final resulting model, containing the 5 domains, and the directions, types and coefficients of the correlation between the variables and domains, was represented using the AMOS /SPSS software, v.22. Upon completion of the specification and identification of the model, a reliability analysis was performed for the domains of the new instrument validated by CFA, which presented a standardized score of 0.93 for the ENS domain, 0.78 for the PMHF-R domain (reformulated PMHF domain), 0.83 for the TS domain, 0.95 for the FC domain and 0.72 for the $\mathrm{VC}$ domain.

Table 3 shows the correlation coefficients between the domains of the theoretical model validated by CFA. It should be noted that only the correlation coefficient between the FC and ENS domains was not statistically significant.

Table 3. Correlation matrix between the domains of the theoretical model

\begin{tabular}{llllll}
\hline DOMAIN & EVAL & PMHF-R & VC & FC & TS \\
\hline ENS & 1 & & & & \\
PMHF-R & $.41^{*}$ & 1 & & & \\
VC & $.40^{*}$ & $.58^{*}$ & 1 & & \\
FC & .10 & $.36^{*}$ & $.17^{*}$ & 1 & \\
TS & $.48^{*}$ & $.68^{*}$ & $.76^{*}$ & $.29^{*}$ & 1 \\
\hline
\end{tabular}

* Correlation is significant at $p<.01$, two-tailed

The highest correlation was found between the VC and TS domains $(r=0.76)$, meaning that $57.76 \%$ of the students who considered the teacher supervision to be positive, also believed that psychiatric nursing provides a valuable contribution to patients, and vice versa. The lowest correlation was identified between the VC and FC domains ( $r=0.17)$, 
meaning that only $2.9 \%$ of the students that thought about pursuing a career in mental health also believed that psychiatric nursing provides a valuable contribution to patients.

The lowest coefficient was found between the FC and VC domains and the highest was between the VC and TS domains. The feeling of being prepared, was also represented as the second highest correlation of the theoretical model $(r=0.68)$ with teacher supervision (TS). That is, when the teacher supervision was considered positive, $46.24 \%$ of the students also felt themselves prepared.

The paired T-test was performed, between the mean scores of the FC and VC domains, at the beginning and end of the mental health disciplines. The magnitude score of Cohen's effect (r) was calculated using the formula $r=\sqrt[2]{\frac{t^{2}}{t^{2+d f}}}$. The negative $\mathrm{T}$ score and significance $(p<.001)$ of the test suggest that there were significant differences in the initial and final means $(t(303)=-5$ and $-4.8 ; p<.001)$, i.e. the $\mathrm{VC}$ Domain score (final) was 0.28 higher than the initial, while the FC Domain score (final) was 0.47 higher than the initial. The Cohen's $r$ obtained was 0.27 , for both domains.

\section{Discussion}

\subsection{Exploratory factor analysis (EFA)}

The authors of the instrument obtained a $57 \%$ variance, with this being $66.07 \%$ in the version analyzed by PCA with 7 components, i.e., a higher value than the original study. Based on the criteria adopted for the performance and interpretation of the factor analysis in the second EFA, items 03, 05, 13, 18, 25 and 29 were removed. According to the author of the instrument, item 29 also presented problems and when it was removed the consistency of POS subscale increased from 0.84 to $0.88 .^{[1]}$

The NS, KMI and VC subscales did not present acceptable values $(<0.70)$ and a more current study ${ }^{[15]}$ recommends removals or improvements in the structure of the instrument, so that its reliability is improved. As the VC subscale score was 0.69 , it was decided to perform a new analysis without the NS and KMI subscales, considering that they presented very low scores. With this, a new analysis of internal consistency was performed using the standardized Cronbach's alpha, with a score of 0.71 being obtained, meaning that the VC subscale could be maintained in the instrument structure.

The structure of the items remained stable, as no significant change occurred in the internal consistency of the instrument, if any other item was removed, except for the possibility of proposing another theoretical model, which would lead to a new reliability analysis. In the original study, ${ }^{[1]}$ the KMI subscale showed a score of 0.56 , higher than the present

Published by Sciedu Press study (0.41), however, all the other subscales showed lower scores, these being 0.51 for the NS subscale, 0.67 for the VC subscale and 0.72 for the PMHF subscale. In the present study, the scores were $0.64,0.95$ and 0.81 , respectively, after the inclusion of item 22 in the PMHF subscale. Finally, the POS subscale, presented a Cronbach's alpha score of 0.84 , compared to 0.89 in the present study.

The convergent validity between the Authoritarianism and Minority Vision domains of the OMI scale, ${ }^{[13]}$ indicates that the scores found among the expected domains, while statistically significant, were not regarded as sufficient to be considered valid for this method, as values greater than 0.45 were expected. ${ }^{[16]}$ Thus, the tested structure of 23 items was not considered valid, through the convergent construct method, since although the correlation exists, it is not sufficiently high, suggesting that they are different constructs. Added to this is the fact that the OMI instrument was not designed for students and dates from the $1980 \mathrm{~s},{ }^{[13]}$ in which the model of psychiatric teaching in hospitals predominated, while at the present time practical education activities in open health services are more frequent. These factors, together, can contribute to reduce the validity of the OMI instrument, even though it has been the only one used in Brazilian studies until now. ${ }^{[17]}$ Regarding the reliability of the domains of the instrument validated by CFA, all presented a satisfactory alpha coefficient, ${ }^{[15]}$ unlike the instrument developed by the author $^{[1]}$ in which several domains did not present acceptable reliability (alpha $<0.70$ ).

\subsection{Confirmatory Factor Analysis (CFA)}

An alternative version to the original was defined, with 23 items, divided into 5 components, to be subjected to this analysis. However, it can be seen that this structure is very different to the original, equivalent to only $59 \%$ of the original instrument, which has 39 items. In the first analysis, some adjustments were necessary, combining the rigor of statistical adjustments with the previous knowledge and experience of the researchers. The changes involved the creation of the ENS and TS domains, addition of items 28 and 22 to the PMHF domain, giving the new name PMHF-R, removal of item 30 and the allocation of correlations between items 26 and 27, 22 and 36, and item 36 with the FC domain. These correlations were admitted since, from the interpretation of the researcher, there were theoretical meanings and relationships between the items, as items 26 (I was well supervised in my clinical internship) and 27 (I felt welcomed by the nursing staff during my clinical internship) belonged to the same original POS domain; ${ }^{[1]}$ item 22 (I felt secure in this psychiatry/mental health clinical internship) links to the same subject as item 36 (I enjoyed my psychiatry/mental 
health internship) and this, with the FC domain, as it can be assumed that, to enjoy the mental health internship has a relationship with the desire of the student to follow a future career in the mental health area. This decision was taken only after the first attempt to confirm the theoretical model. Initially, an "exploration of the data" was performed, before the solidification of the confirmation of the theoretical model. ${ }^{[18-20]}$

The theoretical model based on the original instrument, as well as the instrument itself were shown to be adjusted and approved, since the fit indices used (CFI > 0.9, GFI >0.9, RMSEA < 0.08) indicate such fit. The chi-square model did not appear valid, however, it is understood that due to the sample size being greater than 200 subjects, there is a tendency for failure in the test. ${ }^{[19,20]}$

\subsection{Final considerations}

The aim of evaluating the psychometric properties of the proposed instrument for measuring Nursing undergraduate learning was fulfilled. The original factor structure was not confirmed in Brazil. However, it was possible to develop a theoretical model, based on the factors of the instrument, with two domains being maintained and others reformulated and/or removed. This constructed theoretical model could be tested again in the country of origin (Australia), so that the research and study of these properties can be extended, with the intention of improving the conclusions and advances in the theme of psychiatric nursing teaching and evaluation of nursing students' interest in this area. At the end of the process, the original title of the instrument was maintained, adding the acronym BR, to designate the Brazilian version.

The validation of the original instrument was made in a hybrid way, as important original contributions were introduced, such as the reformulation of domains, the removal of items with low communality and other modifications related to inadequacies that were revealed in the fit of the factor model, giving the instrument better consistency. An explanatory header was also added that did not exist in the original instrument and the pre and post-discipline application methodology was preserved, optionally.

As a novel result, we have an instrument for use in Brazil, among Nursing students in the mental health area, based on another from a different language and culture. It is considered that this study has contributed to the study and teaching of Mental Health Nursing by presenting an instrument for the evaluation of attitudes of students and learning in mental health, applicable in Brazil.

As a study limitation, the lack of reliable and valid instruments on the subject studied is emphasized, which makes it difficult to analyze the validity and perform larger comparisons. The need for future studies to improve the instrument and the resulting theoretical model is highlighted.

\section{CONFlicts of Interest Disclosure}

The authors have no potential conflicts of interest.

\section{REFERENCES}

[1] Happell B, Gouch K. Nursing Students' Attitudes to Mental Health Nursing: Psychometric Properties of a Self-report Scale. Arch Psychiatr Nurs. 2009; 23(5): 376-386. PMid:19766929. http://dx.d oi.org/10.1016/j.apnu.2008.10.005

[2] Happell B, Gaskin CJ. The attitudes of undergraduate nursing students towards mental health nursing: a systematic review. J Clin Nurs. 2012; 22(1-2): 148-58. PMid:23170825. http://dx.doi.org/10. $1111 /$ jocn. 12022

[3] Happell B, Welch T, Moxham L, et al. Keeping the flame alight: understanding and enhancing interest in mental health nursing as a career. Arch Psychiatr Nurs. 2013; 27(4): 161-5. PMid:23915692. http://dx.doi.org/10.1016/j . apnu. 2013.04.002

[4] Stevens J, Brownw G, Graham I. Career in mental health still an unlikely career choice for nursing graduates: a replicated longitudinal study. Int J Ment Health Nurs. 2013; 22(3): 213-20. PMid:22809315. http://dx.doi.org/10.1111/j.1447-0349.2012.00860.x

[5] Sims TT, Overton $S$. The student training and recruitment system. Am J Nurs. 2012; 112(2): 65-8. Available from: http://www. ajno nline. com. Acess on 19 sep 2013.

[6] Pedrão LJ, SA Galera, Silva MC, et al. Attitudes of graduate nursing students towards mental disorders in Brazil, Chile and Peru. Rev
Latino-Am. Enferm. 2005; 13(3): 339-43. http: //dx.doi.org/1 0.1590/S0104-11692005000300008

[7] Avanci RDEC, Malaguti SE, Pedrão LJ. Authoritarianism and benevolence towards mental illness: study with students beginning a nursing program Rev Latino-Am. Enferm. 2002; 19(4): 509-15. http://dx.doi.org/10.1590/S0104-11692002000400007

[8] Mc Ewen M, Wills EM. Theoretical Basis for Nursing. 2.nd edition. USA: Lippincott Williams \& Wilkins; 2007.

[9] Beaton D, Bombardier C, Guillemin F, et al. Guidelines for the process of cross-cultural adaptation of self-report measures. Spine. 2000; 25(24): 3186-91. PMid:11124735. http://dx.doi.org/10.1097 100007632-200012150-00014

[10] Soares MH, Luís MAV, Hirata AGP. Cross-cultural cultural adaptation of the "Nursing Students' Attitudes Toward Mental Health Nursing and Consumers" in Brazil. Rev Bras Enferm. 2015; 68(2): 198205. http://dx.doi.org/10.1590/0034-7167.2015680203i

[11] Reichenheim ME, Moraes CL. Operationalizing the cross-cultural adaptation of epidemiological measurement instruments. Rev Saúde Públ. 2007; 41(4): 665-73. PMid:17589768. http://dx.doi .org /10.1590/S0034-89102006005000035

[12] Cohen J, Struening EL. Opinions about mental illness in the personnel of two large mental hospitals. J Abn Soc Psychol. 1962; 64(5): 
349-360. http://dx.doi.org/10.1037/h0045526

[13] Rodrigues CRC. Attitudes towards mental illness: cross-sectional study of a sample of health professionals. Ribeirão Preto, 1983. Thesis (Doctoral) - Faculdade de Medicina de Ribeirão Preto, Universidade de São Paulo.

[14] Byrne BM. Structural Equation Modeling with AMOS: basic concepts, application and programming. 2nd. Ed. New York: Routledge Taylor \& Francis Group; 2010.

[15] Maroco J, Garcia-Marques T. Qual a fiabilidade do alfa de Cronbach? Questões antigas e soluções modernas? Lab Psicol. 2006; 4(1): 65-90.

[16] De VON HA, et al. A psychometric toolbox for testing validity and reliability. J Nurs Schol. 2007; 39(2): 155-64. http://dx.doi.o $\mathrm{rg} / 10.1111 / \mathrm{j} \cdot 1547-5069 \cdot 2007.00161 . \mathrm{x}$

[17] Pedrão LJ, Avanci RC, Malaguti SE, et al. Attitudes towards mental health: a comparative study between nursing freshmen and seniors. Medic. 2003; 36(1): 37-44. http://dx.doi.org/10.11606/is sn. 2176-7262.v36i1p37-44

[18] Hair JF, Anderson RE, Tatham RL, et al. Multivariate data analysis. 5th. ed. New Jersey: Prentice Hall; 1998.

[19] Brown TA. Confirmatory factor analysis for apllied research. NY: The Guilford Press, 2006. 475 p.

[20] Kline RB. Principles and practice of structural equation modeling. 3rd. ed. NY: The Guilford Press; 2011. 427 p. 